\title{
Critical area for memory decline after mesial temporal resection in epilepsy patients
}

\author{
Dahye Kim, BS, ${ }^{1}$ June Sic Kim, PhD, ${ }^{2}$ Woorim Jeong, PhD, ${ }^{3,4}$ Min-Sup Shin, PhD, ${ }^{5}$ and \\ Chun Kee Chung, MD, PhD1,3,4
}

\begin{abstract}
1Department of Brain and Cognitive Sciences and ${ }^{2}$ Research Institute of Basic Sciences, Seoul National University College of Natural Sciences; ${ }^{3}$ Department of Neurosurgery, Seoul National University Hospital; and ${ }^{4}$ Neuroscience Research Institute and ${ }^{5}$ Department of Psychiatry and Behavioral Science, Seoul National University College of Medicine, Seoul, Republic of Korea
\end{abstract}

OBJECTIVE Mesial temporal lobe epilepsy (MTLE) surgery is associated with a risk of memory decline after surgery, but the effect of the extent and locus of temporal resection on postoperative memory function are controversial. The authors' aim in this study was to confirm if selective resection is effective in preserving memory function and identify critical areas for specific memory decline after temporal resection.

METHODS In this single-center retrospective study, the authors investigated data from patients who underwent unilateral MTLE surgery between 2005 and 2015. Data from 74 MTLE patients (60.8\% of whom were female; mean [SD] age at surgery 32 years [ 8.91 years] and duration of epilepsy 16 years [9.65 years]) with histologically proven hippocampal sclerosis were included. Forty-two patients underwent left-sided surgery. The resection area was manually delineated on each patient's postoperative T1-weighted images. Mapping was performed to see if the resected group, compared with the nonresected group, had worse postoperative memory in various memory domains, including verbal item, verbal associative, and figural memory.

RESULTS Overall, $95.9 \%$ had a favorable epilepsy outcome. In verbal item memory, resection of the left lateral temporal area was related to postoperative decline in immediate and delayed recall scores of word lists. In verbal associative memory, resection of the anterior part of the left hippocampus, left parahippocampal area, and left lateral temporal area was related to postoperative decline in immediate recall scores of word pairs. Resection of the posterior part of the left hippocampus, left parahippocampal area, and left lateral temporal area was related to delayed recall scores of the same task. Similarly, in the figural memory, postoperative decline of immediate recall scores was associated with the resection of the anterior part of the right hippocampus, amygdala, parahippocampal area, and superior temporal area, and decline of delayed recall scores was related to resection of the posterior part of the right hippocampus and parahippocampal area.

CONCLUSIONS Using voxel-based analysis, which accounts for the individual differences in the resection, the authors found a critical region for postoperative memory decline that is not revealed in the region-of-interest or groupwise comparison. Particularly, resection of the hippocampus was related to associative memory. In both verbal and visual memory, resection of the anterior part of the hippocampus was associated with immediate recall, and resection of the posterior part of the hippocampus was associated with delayed recall. Therefore, the authors' results suggest that selective resection may be effective in preserving postoperative memory decline.

https://thejns.org/doi/abs/10.3171/2019.10.JNS191932

KEYWORDS temporal lobe epilepsy; epilepsy surgery; memory outcome; hippocampus; medial temporal lobe

$\mathrm{R}$ ESECTIVE surgery for mesial temporal lobe epilepsy (MTLE) has a risk of memory decline after surgery, since the mesial temporal area plays a pivotal role in memory functions..$^{36}$ Approximately $30 \%-60 \%$ of patients experience postoperative verbal memory decline after left anterior temporal resection. ${ }^{30}$ Also, patients af- ter right-sided surgery have a risk of decline in nonverbal memory, although it is not as consistent as in verbal memory. ${ }^{6,39}$ Although more selective resections have been proposed to minimize postoperative memory decline, ${ }^{35}$ whether selective resection is actually effective for preserving memory function remains controversial. ${ }^{15,29}$ To

ABBREVIATIONS AAL = Automated Anatomical Learning; $\mathrm{AH}=$ amygdalohippocampectomy; $\mathrm{ATL}=$ anterior temporal lobectomy; $\mathrm{MTLE}=$ mesial temporal lobe epilepsy; $\mathrm{ROI}=$ region of interest; $\mathrm{SAH}=$ selective $\mathrm{AH} ; \mathrm{TPR}=$ temporal pole resection.

SUBMITTED July 15, 2019. ACCEPTED October 25, 2019.

INCLUDE WHEN CITING Published online January 3, 2020; DOI: 10.3171/2019.10.JNS191932. 
make things worse, if it is effective, which area in the temporal lobe is critical for postoperative memory decline also remains controversial. ${ }^{1,32}$ These controversies may be due to the limitation of groupwise comparison analyses, which have included neglect of individual differences in extent and in the exact locus of resection and usually comparisons of postoperative memory change according to the different surgical procedures.

Furthermore, although each temporal area is critical for specific memory subfunctions ${ }^{28,42}$ only a few studies have investigated the relationship between memory outcome and resection of specific temporal areas in a way that accounts for individual differences in the extent and locus of temporal resection..$^{20,24,33}$ These studies compared resected areas based on region-of-interest (ROI) analysis at the individual level. However, direct comparison between these studies is difficult, since the atlas or segmentation methods used by ROI-based analyses were different.

We hypothesized that resection of specific areas would decrease specific subfunctions of memory postoperatively. Therefore, our aim was to confirm if selective resection is actually effective for preserving memory function and to identify critical areas for specific memory decline after temporal resection by analyzing each patient's MRI studies in an atlas-free, voxel-based way.

\section{Methods \\ Patients}

We retrospectively included patients with MTLE who underwent resective surgery between 2005 and 2015 in the epilepsy clinic at the Seoul National University Hospital. Each patient's surgery was performed based on comprehensive preoperative tests, including history and neurological examination, seizure semiology, long-term video-EEG monitoring, structural MRI, PET, interictal and ictal SPECT, invasive monitoring if necessary, and/ or magnetoencephalography. Inclusion criteria were intractable MTLE patients with histologically proven hippocampal sclerosis, whose postoperative high-resolution MRI and pre- and postoperative neuropsychological test results were available. To control other confounding factors caused by differences in clinical practice, only patients who had surgery by a single neurosurgeon (C.K.C.) were included. Finally, we included 74 patients $(60.8 \%$ female; 42 left-sided surgeries) in this study.

All patients underwent either a temporal pole resection with an amygdalohippocampectomy (TPR+AH) or a selective amygdalohippocampectomy (SAH). The type of surgery was randomly assigned to the patients whose epileptogenic zone was located in the medial temporal lobe determined by preoperative evaluation. In TPR+AH, the patients underwent a TPR (the average extent of the resection was $3.39 \pm 0.78 \mathrm{~cm}$ from the temporal pole) with an $\mathrm{AH} .{ }^{13}$ For $\mathrm{SAH}$, all patients underwent a transsylvian approach, entering the temporal horn of the lateral ventricle through the temporal stem. To determine seizure outcome, we used the Engel Epilepsy Surgery Outcome Scale (class I, free of disabling seizure; class II, rare disabling seizures; class III, worthwhile improvement; and class IV, no worthwhile improvement). ${ }^{8}$ All patients were in Engel classes I
TABLE 1. Demographics and clinical characteristics of patients

\begin{tabular}{|c|c|c|}
\hline & Lt MTLE $(n=42)$ & Rt MTLE $(n=32)$ \\
\hline Sex, M/F & $16: 26$ & $13: 19$ \\
\hline \multicolumn{3}{|l|}{ Handedness, n (\%) } \\
\hline $\mathrm{Rt}$ & $37(88.1)$ & $30(93.8)$ \\
\hline $\mathrm{Lt}$ & $4(9.5)$ & $1(3.1)$ \\
\hline Ambidextrous & $1(2.4)$ & $1(3.1)$ \\
\hline \multicolumn{3}{|l|}{ Surgery type, n (\%) } \\
\hline SAH & $14(33.3)$ & $8(25.0)$ \\
\hline $\mathrm{TPR}+\mathrm{AH}$ & $28(66.7)$ & $24(75.0)$ \\
\hline $\begin{array}{l}\text { Mean age at seizure onset, } \\
\text { yrs }\end{array}$ & $15.8(8.5)$ & $18.3(12.3)$ \\
\hline Mean duration of illness, yrs & $16.4(9.5)$ & $14.5(10.1)$ \\
\hline Mean age at surgery, yrs & $32.3(8.2)$ & $32.8(10.0)$ \\
\hline \multicolumn{3}{|l|}{ Engel class, $\mathrm{n}(\%)$} \\
\hline I & $39(92.9)$ & $29(90.6)$ \\
\hline II & $2(4.8)$ & $1(3.1)$ \\
\hline III & $1(2.4)$ & $2(6.3)$ \\
\hline
\end{tabular}

Mean values are presented as the mean (SD).

to III; no patient was in class IV. This study was approved by the institutional review board of the Seoul National University Hospital Clinical Research Institute. A summary of patient demographics is provided in Table 1 .

\section{Neuropsychological Memory Test}

The same comprehensive neuropsychological assessments were conducted both before surgery (within 1 month) and after surgery as part of routine clinical practice. ${ }^{31}$ Verbal item memory was assessed using the verbal immediate and delayed recall subtests from the Korean version of the Rey Auditory Verbal Learning Test. This test requires immediate recall for a list of 15 words presented audibly at intervals of 1 second and repeated 5 times (verbal item immediate recall) and after 20 minutes requires recall for the list of words (verbal item delayed recall). Verbal associative memory was assessed using the verbal paired-associates subtest from the Wechsler Memory Scale-third edition, which requires patients to learn 14 pairs of words presented audibly, to then listen to the first word of each pair, and to recall immediately the other word in the pair (verbal associative immediate recall); after 30 minutes, the first word of the pair was presented and the patient was required to recall the other word in the pair (verbal associative delayed recall). Nonverbal memory was assessed using scores of the Korean version of the Rey Complex Figure Test, which requires patients to reproduce a complicated line drawing, first by copying it immediately (figural immediate recall) and then drawing it from memory after 20 minutes (figural delayed recall). In order to account for these individual differences in changes of memory functions, the postoperative memory change was calculated by subtracting the presurgical scores from the postsurgical scores of the patient.

\section{Image Analysis}

T1-weighted 3D spoiled-gradient MR images with 


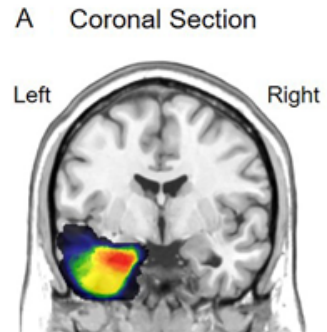

$Y=123$

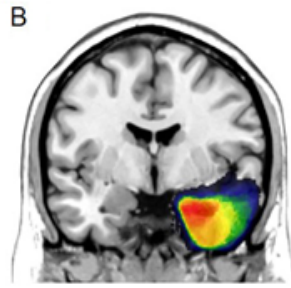

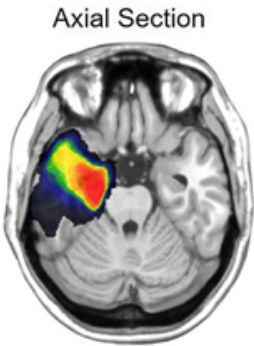

$Z=48$

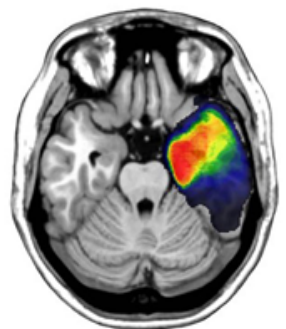

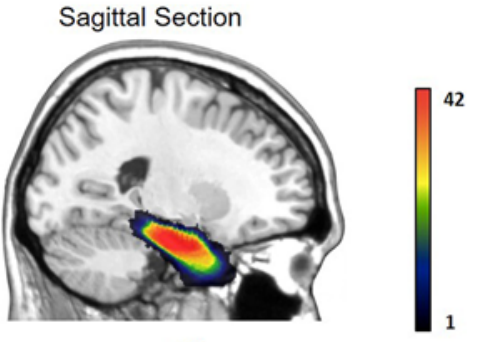

$\mathrm{x}=68$

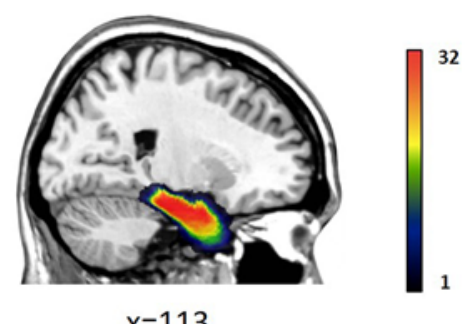

$x=113$

FIG. 1. Overlapped images of manually delineated individual resection maps. A: Frequency of the resected area in left-sided MTLE surgery. B: Frequency of the resected area in right-sided MTLE surgery. For each voxel, the frequency of patients who had the resection are represented by colors. As shown in the scale bar, red represents the area resected in most patients, and blue represents the area resected less frequently. Figure is available in color online only.

a thickness of $1.0 \mathrm{~mm}$ were used. All patients were examined using a 1.5T (Signa Horizon, GE Healthcare; or Magnetom Avanto, Siemens) or a 3T (Signa Excite, GE Healthcare; or Magnetom Verio, Siemens) MRI system both before and after surgery. The mean follow-up for postoperative MRI was $1.10 \pm 2.23$ years. Lesions were manually delineated by one author (D.H.K.) on native T1weighted images using MRIcron software (http://www. nitrc.org/projects/mricron/). Then, the T1-weighted images were segmented and normalized into the Montreal Neurological Institute (MNI) template by using the Clinical Toolbox ${ }^{3,27}$ for SPM12 (https://www.fil.ion.ucl.ac.uk/ software/spm12/), which implements a unified nonlinear normalization and segmentation process. Normalization was conducted using lesion cost function masking ${ }^{3,27}$ to prevent image distortion, which is often observed in brain images with lesions.

All manually delineated individual resection maps were overlapped on the MNI atlas for generating the resection frequency map in order to display individual differences in extent and locus of resection and for voxel-based statistical analysis (Fig. 1)..$^{2,9,18}$ To find the area critical for postoperative memory decline, we performed two forms of analysis: 1) voxel-based analysis that accounted for individual differences in extent and locus of resection; and 2) ROI analysis, which needed segmentation of the resection area using the Automated Anatomical Learning (AAL) atlas and investigated the correlation between resection volume of each temporal area and memory test score changes. The ROI-based analysis was performed to compare our results with those of previous ROI-based studies.

\section{Statistical Analysis \\ Voxel-Based Analyses}

In this study, according to the material-specific memo- ry model ${ }^{40}$ we analyzed verbal memory in the left MTLE group and nonverbal memory in the right MTLE group. For each voxel, patients were divided into two groups depending on whether the voxel was resected or not. The difference in postoperative memory score changes between the two groups was tested using the Mann-Whitney U-test. Finally, mapping was performed on voxels with a significantly small value of memory score change in the resected group compared with the nonresected group $(\mathrm{p}<$ 0.05 , familywise error corrected; cluster extent threshold, 30 voxels). All voxel-based statistical analyses were performed using Matlab (version R2018a, MathWorks Corp.).

\section{ROI-Based Analyses}

The resection map of each patient was segmented into subareas using the modified AAL atlas (Fig. 2). Because the entorhinal area is known to be important for memory functions, we further subdivided the parahippocampal area of the existing AAL atlas into entorhinal and parahippocampal areas ${ }^{19}$ using MRIcron and Matlab. Finally, the temporal area was segmented into the hippocampus, entorhinal, parahippocampal, fusiform, amygdala, superior temporal, middle temporal, and inferior temporal areas. We calculated the resection percentage of each temporal ROI for each patient using Matlab.

At first, a univariate analysis was performed for the correlation between the resection volume of each temporal structure (ROI) and the score change of each memory test. The $p$ values of the correlation coefficients were adjusted using Bonferroni corrections for multiple-region comparison (8 ROIs). Second, multivariate linear regression analysis was performed with the memory change score as the dependent variable and the resected volumes of each ROI as the independent variables. With the multivariate linear regression analysis, it was possible to see which area was related to the change in memory while correcting for the 


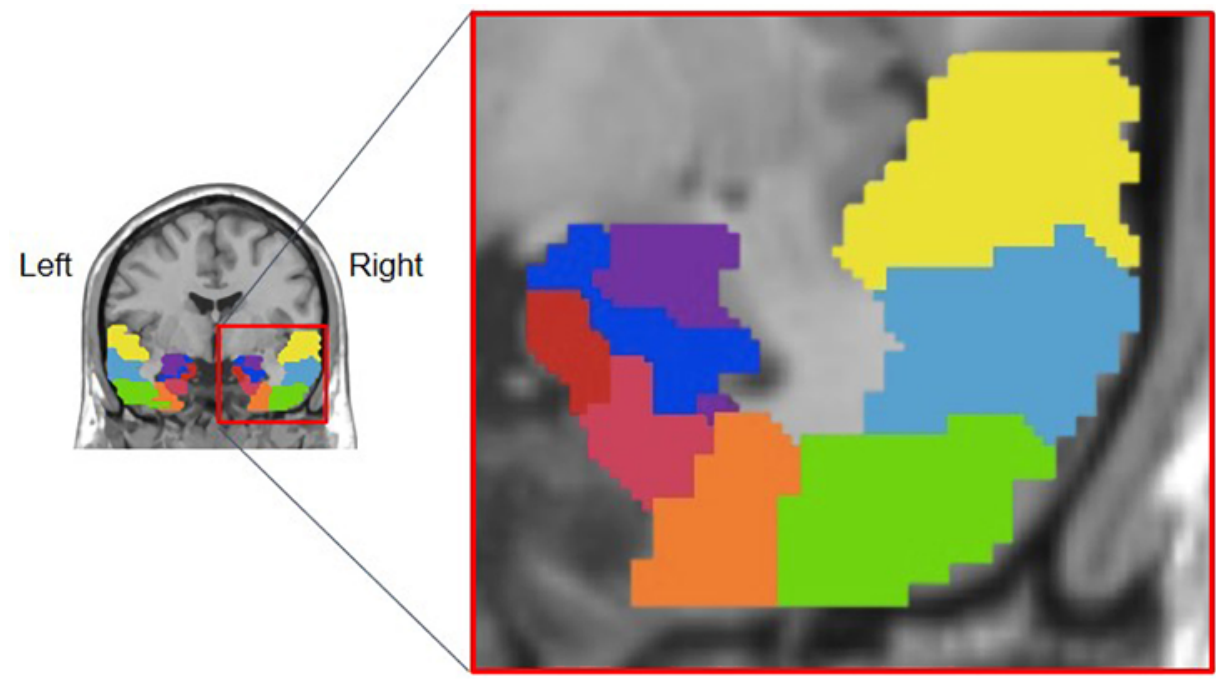

- Hippocampus

- Amygdala

Entorhinal

Parahippocampal

Superior Temporal

Middle Temporal

Inferior Temporal

Fusiform

FIG. 2. Segmentation of the temporal area using the modified AAL atlas. For ROI-based analysis, the temporal area was segmented into the hippocampus, amygdala, entorhinal cortex, parahippocampal area, superior temporal area, middle temporal area, inferior temporal area, and fusiform area. The parahippocampal area of the AAL atlas was subdivided into the entorhinal and parahippocampal areas. Figure is available in color online only.

effect of the resection of the other areas. The model was constructed by a backward elimination, which begins with a model that includes all potential predictors and successively drops one at a time if it does not contribute to the regression equation. The $\mathrm{p}$ values of the coefficients were adjusted with Bonferroni corrections for multiple-region comparison (8 ROIs). In the ROI-based analyses, all statistical analyses were performed using IBM SPSS (version 25, IBM Corp.).

\section{Results}

\section{Surgical Outcome}

The mean follow-up for seizure outcome was $6.25 \pm$ 2.91 years. Because most of our patients achieved favor-

TABLE 2. Results of preoperative and postoperative neuropsychological memory testing

\begin{tabular}{lcc}
\hline & Preop & Postop \\
\hline Lt MTLE & & \\
\hline K-RAVLT $(\mathrm{n}=42)$ & & \\
\hline Verbal item immediate recall & $39.8(8.7)$ & $39.4(10.6)$ \\
\hline Verbal item delayed recall & $5.9(3.2)$ & $6.8(3.7)$ \\
\hline WMS-III ( $=36)$ & & \\
\hline Verbal associative immediate recall & $6.4(3.1)$ & $6.4(3.4)$ \\
\hline Verbal associative delayed recall & $6.0(3.1)$ & $5.9(3.5)$ \\
\hline Rt MTLE & & \\
\hline K-RCFT ( $=32)$ & & \\
\hline Figural immediate recall & $16.2(7.6)$ & $16.6(5.7)$ \\
\hline Figural delayed recall & $15.1(7.5)$ & $16.0(6.6)$ \\
\hline K-RAVT = Kor
\end{tabular}

K-RAVLT = Korean version of the Rey Auditory Verbal Learning Test; K-RCFT $=$ Korean version of the Rey Complex Figure Test; WMS-III = Wechsler Memory Scale-third edition.

Values are presented as the mean (SD). able seizure outcomes (class I or II, 96\%), we could not investigate the differences in seizure outcome in terms of the procedure (SAH or TPR+AH). There were no significant differences in duration of illness $(p=0.546)$, age at surgery $(\mathrm{p}=0.918)$, or age at seizure onset $(\mathrm{p}=$ 0.643 ) between patients undergoing left MTLE and those undergoing right MTLE surgery. Nine left (21.4\%) and 6 right (18.8\%) MTLE patients stopped taking antiepileptic drugs completely after surgery, and all remaining patients took less medication after surgery; no patient had an increase in the number of antiepileptic drugs after surgery.

\section{Neurosurgical Outcome}

The mean interval between pre- and postoperative neuropsychological tests was $1.61 \pm 1.18$ years. Details of the neuropsychological memory scores are presented in Table 2. There were no significant differences in group-level comparison of pre- and postoperative memory by pairedsample t-test. However, there were various differences in the individual level as shown in Fig. 3.

\section{Voxel-Based Statistical Mapping of Verbal Memory}

For verbal item memory, resection of the left lateral temporal area (superior, middle, inferior temporal, and fusiform) was significantly associated with the decline in immediate and delayed recall of a word list (Fig. 4). For the verbal associative memory, resection of the anterior part of the left hippocampus, left parahippocampal area, and left lateral temporal area (superior, middle, inferior temporal, and fusiform) was significantly related to a worse memory outcome in immediate recall of word pairs. Resection of the posterior part of the left hippocampus, left parahippocampal area, and left lateral temporal area (superior, middle, inferior, and fusiform) was significantly related to a worse memory outcome in delayed recall of word pairs. 

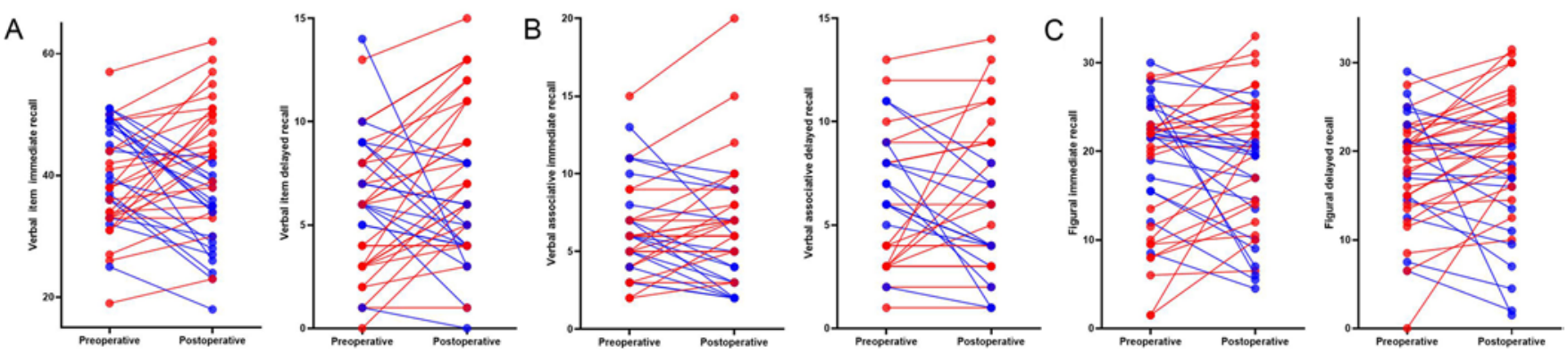

FIG. 3. Individual memory changes in verbal item memory (A), verbal associative memory (B), and figural memory (C). Each line represents an individual patient. Red lines indicate postoperative improvement and blue lines represent postoperative decline.

Figure is available in color online only.

\section{Voxel-Based Statistical Mapping of Nonverbal Memory}

In the nonverbal memory, resection of the anterior part of the right hippocampus, right amygdala, right parahippocampal area, and medial part of the right superior temporal area was significantly associated with the decline of immediate recall of complex figures. Resection of the posterior part of the right hippocampus and the right parahippocampal area was significantly associated with worse outcome in delayed recall for the same task (Fig. 5).

\section{ROI-Based Analysis}

In the univariate analysis, the change in the verbal item delayed recall score was negatively correlated with the resected volume of the left superior temporal $(r=-0.426, p$ $<0.05$, corrected) and left inferior temporal $(\mathrm{r}=-0.420$, $\mathrm{p}<0.05$, corrected) areas. For the multivariate linear regression, the change in the verbal item delayed recall score was negatively correlated with the resected volume of the left inferior temporal area $(\beta=-0.50, p<0.01$, corrected), and the change in the verbal associative immediate recall score was negatively correlated with the resected volume of the left hippocampus $(\beta=-0.71, \mathrm{p}<0.05$, corrected $)$ and left middle temporal area $(\beta=-1.13, \mathrm{p}<0.05$, corrected) (Table 3).

Through the multivariate analysis that investigated the effect of the resection of each ROI while correcting for the effect of the resection of the other areas, we show that the resected volume of the hippocampus was related to the change in associative memory.

\section{Group-Level Analysis}

We compared the postoperative memory changes according to surgery type (i.e., SAH or TPR+AH), but there were no significant differences. Interestingly, although there was no significant group-level comparison difference, the total volume of the resection was correlated with the change in the verbal item delayed recall score $(r=$ $-0.40, \mathrm{p}<0.05$, corrected).

\section{Discussion}

Our aim in this study was to confirm if selective resection is actually effective for preserving memory function and to identify critical areas for specific memory decline after temporal resection. We investigated each patient's
MRI studies using a voxel-based analysis to account for individual differences in the extent and locus of resection in the temporal structures.

\section{Critical Area for Postoperative Verbal Memory Decline}

From voxel-based analysis, we found that resection of the left lateral temporal area was related to worse outcome of postoperative verbal item memory, and resection of the left hippocampus, left parahippocampal area, and left lateral temporal area was related to postoperative verbal associative memory decline. Interestingly, in associative memory, resection of the anterior part of the left hippocampus was associated with worse immediate recall, whereas resection of the posterior part of the left hippocampus was associated with worse delayed recall.

In summary, there was a task specificity in verbal memory that suggests it is affected differently by resection of each left temporal area. There was no statistical significance in the extent of resection of the hippocampus when observed with the item memory task, but there was a significant relationship between the resection of the hippocampus and postoperative memory change in the task requiring associative memory. Also, the locus of the mapped hippocampal area was dependent on the retention interval.

From the task-specific perspective in verbal memory, several previous studies reported dissociation of item and associative memory. Verbal item memory function declined after left standard anterior temporal lobectomy (ATL), but not after SAH, which preserves the lateral temporal area.$^{12}$ Furthermore, a larger extent of resection in the superior temporal area resulted in a worse postoperative verbal item memory change. ${ }^{1}$ In a bilateral hippocampal lesion study, patients did worse on the associative memory task than on the item memory tasks. ${ }^{11}$ In a functional MRI study, the hippocampus was more activated in memory for the associations between informational elements than in memory for individual elements. ${ }^{10}$ Therefore, it is quite reasonable that the relationship between postoperative memory decline and the extent of hippocampal resection may be found only after considering task specificity.

\section{Critical Area for Postoperative Nonverbal Memory Decline}

For figural memory, we found that resection of the anterior part of the right hippocampus, right amygdala, right 
A

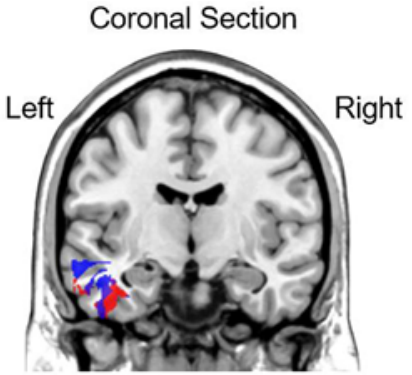

$y=116$

B

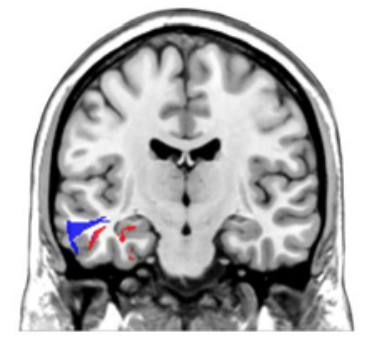

$y=111$

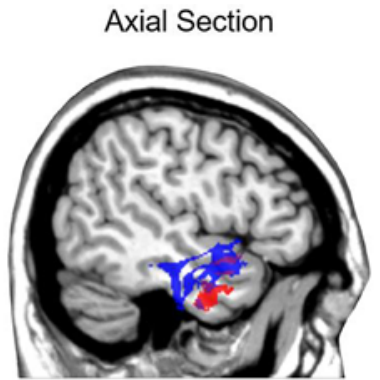

$x=41$

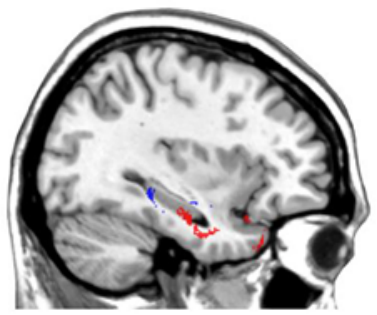

$x=57$

Immediate Recall

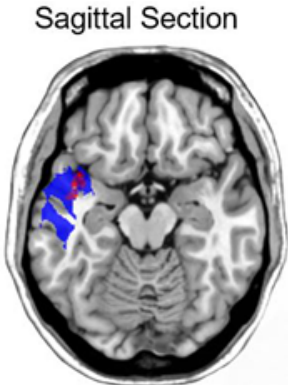

$z=56$

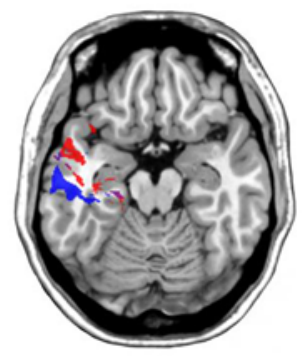

$z=54$

Delayed Recall

FIG. 4. Voxel-based statistical mapping of verbal memory. Mapping was performed on voxels with a significantly small value of memory score change in the resected group compared with the nonresected group $(p<0.05$, familywise error corrected; cluster extent threshold, 30 voxels). A: Voxel-based statistical mapping of verbal item memory. B: Voxel-based statistical mapping of verbal associative memory. Figure is available in color online only.

parahippocampal area, and medial part of the right superior temporal area was related to the decline in immediate recall of complex figures. On the other hand, the decline in the figural delayed recall was related to the resection of the posterior part of the right hippocampus and right parahippocampal area. Several previous studies have investigated the relationship between the right temporal resection and nonverbal memory performance (for a review, see the article by $\operatorname{Vaz}^{39}$ ). In a previous study, the extent of right hippocampal resection was correlated with worse visuospatial memory. ${ }^{25}$ Another study found that the patients who underwent right-sided surgery showed a decline in postoperative figural learning. ${ }^{13}$ However, many other previous studies have failed to find a significant relationship between the resection of the right temporal area and postoperative decline in nonverbal memory functions. ${ }^{16,38,39}$

Considering our results, the locus of hippocampal resection may affect immediate and delayed recall differently. Interestingly, we also found similar results in verbal associative memory. Resection of the anterior part of the hippocampus was associated with a worse immediate recall outcome, whereas resection of the posterior part of the hippocampus was associated with a worse delayed recall outcome. There are many studies about the functional dif-

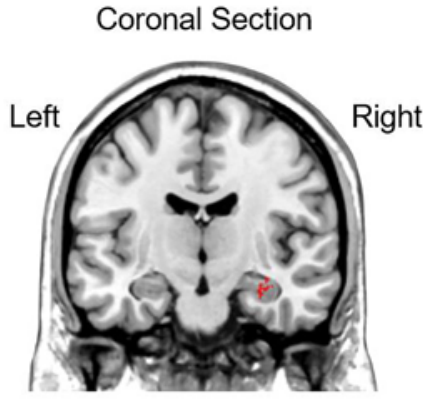

$y=113$

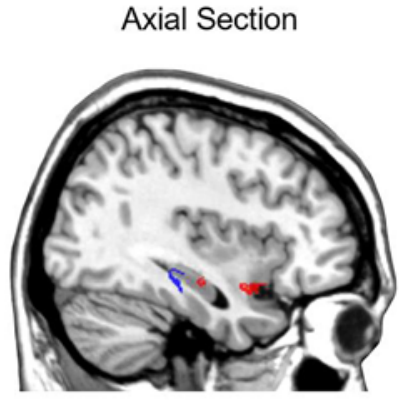

$x=57$

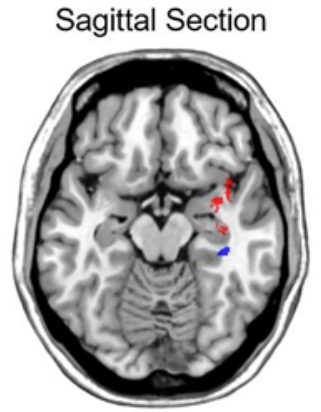

$z=57$

Immediate Recall

Delayed Recall

FIG. 5. Voxel-based statistical mapping of figural memory. Figure is available in color online only. 
TABLE 3. Regression coefficients for variables that remained after backward elimination in a multiple linear regression predicting memory score change

\begin{tabular}{llccccc}
\hline \multicolumn{1}{c}{ Dependent Variable } & Predictor Variable & Standardized $\beta$ & Standardized Error & $95 \% \mathrm{Cl}$ & $\mathrm{t}$ & $\mathrm{p}$ Value \\
\hline Verbal item delayed recall $(\mathrm{n}=42)$ & Entorhinal & 0.31 & 0.066 & $(0.013$ to 0.279$)$ & 2.216 & 0.260 \\
\cline { 2 - 6 } & Inferior temporal & -0.50 & 0.049 & $(-0.270$ to -0.073$)$ & -3.528 & $0.009^{*}$ \\
\hline Verbal associative immediate recall $(\mathrm{n}=36)$ & Hippocampus & -0.71 & 0.062 & $(-0.317$ to -0.066$)$ & -3.107 & $0.032^{*}$ \\
\cline { 2 - 6 } & Amygdala & 0.39 & 0.056 & $(-0.010$ to 0.220$)$ & 1.871 & 0.566 \\
\cline { 2 - 6 } & Fusiform & 0.70 & 0.092 & $(-0.011$ to -0.363$)$ & 1.919 & 0.513 \\
\cline { 2 - 7 } & Middle temporal & -1.13 & 0.034 & $(-0.175$ to -0.036$)$ & -3.095 & $0.033^{*}$ \\
\hline
\end{tabular}

* Significant after correction for multiple comparison.

ferentiation along the anteroposterior axis of the hippocampus. In line with our findings, a nonhuman primate study reported that posterior hippocampal neurons were predominantly activated compared with anterior hippocampal neurons in a delayed spatial memory task. ${ }^{4}$ Similarly, in a functional MRI study in humans, anterior hippocampal activity decreased over the retention interval; however, posterior hippocampal activity did not decline over time..$^{5}$ In this sense, we speculate that the anterior part of the hippocampus might play an important role for immediate recall, and the posterior part of the hippocampus has an important role for delayed recall. Therefore, we suggest that specific memory types (i.e., immediate or delayed) along with materials (i.e., verbal or nonverbal) should also be considered in predicting memory outcome after resective surgery.

From the task-specific perspective in figural memory, several nonhuman primate studies reported that the hippocampal lesion impaired visual paired comparison tasks (i.e., visual associative memory task) but not visual item memory tasks. ${ }^{23,26}$ However, there is no study in humans that has clarified the task specificity of nonverbal memory decline in patients who underwent temporal resection. Therefore, future study is warranted to test the task specificity in the nonverbal memory by using various figural memory tests after resection of the right temporal area in humans.

\section{Strength of Voxel-Based Analysis}

Because memory decline is the main issue with temporal resection, more conservative and less-extensive resections have been proposed to theoretically minimize postoperative memory deficits. Hence, SAH has emerged as an alternative to standard ATL. ${ }^{35}$ There have been inconsistencies in studies on the actual effect of conservative surgery to preserve postoperative memory function. Some studies have argued that a larger and more posterior medial resection was not associated with worse verbal memory outcome, ${ }^{17,32,38,41}$ but others have insisted that the outcome in verbal learning and memory was poorer after larger resections. ${ }^{1,14,21}$ Likewise, a decline in nonverbal memory after right temporal resection has been reported, but there are discrepancies between the results. ${ }^{38,39}$ One reason for these controversial results may be that previous studies used a groupwise comparison. Most of the studies focused on comparing the results of the two different surgical types. ${ }^{13,15,22,32,37}$ However, even if the same surgical procedure was performed, there may be individual differences in the actual resection extent and area resected. Likewise, for additional group-level analyses in our data, there was no significant difference in postoperative memory changes depending on the type of surgery (i.e., SAH or TPR+AH) in all memory subdomains. Interestingly, although there was no significant group-level comparison result, the total volume of resection was correlated with the change in the verbal item delayed recall score $(r=-0.40, p<0.05$, corrected for multiple comparisons). With group-level analysis, it is difficult to take into account the differences in extent and locus of resection between individuals in detail. Similarly, in our study, there was no significant result with the ROI-based analysis in figural memory; the significant result was obtained only with the voxel-based analysis, probably because the latter analysis reflected more sensitively the individual differences that cannot be accounted for in the ROI-based analysis and thus arrived at significant results. Through our two types of analysis on the same data, we suggest that it is important to account for individual differences in studies investigating postoperative cognitive outcome. Therefore, voxel-based analysis, which can account for the individual differences in resection, will be useful in defining the critical area for postoperative memory changes and could provide a resolution to the controversy over whether selective resection is effective in postoperative cognitive outcome.

\section{Limitations}

There are several caveats that should be taken into consideration when generalizing our findings on the critical area for specific memory decline postoperatively. First, statistical analysis cannot be performed on voxels that have not been resected in any patient or resected in all patients. Second, although each voxel and ROI are statistically processed independently in this study, it remains unclear whether the adjacent area is truly independent in resection. Third, since we used only one visual memory test, we could not observe the difference between the item and the associative memory in the nonverbal domain. Fourth, we did not see an individual difference in preoperative hippocampal volumes. Finally, there are claims that memory change may depend on the extent of white matter disconnection. ${ }^{7,34}$ Further studies taking the disconnection of white matter into account are needed. 


\section{Conclusions}

This is the first study to investigate the effect of temporal resection on postoperative memory decline by employing voxel-based analysis, which accounts for individual differences in the extent and locus of resection. We found that resection of specific areas impairs corresponding specific subdomains of memory. In particular, resection of the hippocampus was related to associative memory. In both verbal memory and visual memory, resection of the anterior part of the hippocampus was associated with immediate recall, and resection of the posterior part of the hippocampus was associated with delayed recall. Therefore, our results suggest that selective resection may be effective in preserving postoperative memory decline.

\section{Acknowledgments}

We would like to express special thanks for help in data acquisition to Sang Kun Lee, M.D., head of the Epilepsy Monitoring Unit at Seoul National University Hospital and to Bohee Lee for help in proofreading. This research was supported by grants (2018M3C7A1022317, 2019R1A2C1009674, 2016M3C7A1904984) from the Basic Science Research Program through the National Research Foundation (NRF), funded by the Ministry of Science \& ICT, South Korea.

\section{References}

1. Alpherts WC, Vermeulen J, van Rijen PC, da Silva FH, van Veelen CW: Standard versus tailored left temporal lobe resections: differences in cognitive outcome? Neuropsychologia 46:455-460, 2008

2. Bonilha L, Yasuda CL, Rorden C, Li LM, Tedeschi H, de Oliveira E, et al: Does resection of the medial temporal lobe improve the outcome of temporal lobe epilepsy surgery? Epilepsia 48:571-578, 2007

3. Brett M, Leff AP, Rorden C, Ashburner J: Spatial normalization of brain images with focal lesions using cost function masking. Neuroimage 14:486-500, 2001

4. Colombo M, Fernandez T, Nakamura K, Gross CG: Functional differentiation along the anterior-posterior axis of the hippocampus in monkeys. J Neurophysiol 80:1002-1005, 1998

5. Dandolo LC, Schwabe L: Time-dependent memory transformation along the hippocampal anterior-posterior axis. Nat Commun 9:1205, 2018

6. Dulay MF, Levin HS, York MK, Mizrahi EM, Verma A, Goldsmith I, et al: Predictors of individual visual memory decline after unilateral anterior temporal lobe resection. Neurology 72:1837-1842, 2009

7. Elliott CA, Gross DW, Wheatley BM, Beaulieu C, Sankar T: Longitudinal hippocampal and extra-hippocampal microstructural and macrostructural changes following temporal lobe epilepsy surgery. Epilepsy Res 140:128-137, 2018

8. Engel JJ (ed): Outcome With Respect to Epileptic Seizures, ed 2. New York: Raven Press, 1993

9. Galovic M, Baudracco I, Wright-Goff E, Pillajo G, Nachev P, Wandschneider B, et al: Association of piriform cortex resection with surgical outcomes in patients with temporal lobe epilepsy. JAMA Neurol 76:690-700, 2019

10. Giovanello KS, Schnyer DM, Verfaellie M: A critical role for the anterior hippocampus in relational memory: evidence from an fMRI study comparing associative and item recognition. Hippocampus 14:5-8, 2004

11. Gold JJ, Hopkins RO, Squire LR: Single-item memory, associative memory, and the human hippocampus. Learn Mem 13:644-649, 2006
12. Helmstaedter C, Grunwald T, Lehnertz K, Gleissner U, Elger CE: Differential involvement of left temporolateral and temporomesial structures in verbal declarative learning and memory: evidence from temporal lobe epilepsy. Brain Cogn 35:110-131, 1997

13. Helmstaedter C, Richter S, Röske S, Oltmanns F, Schramm J, Lehmann TN: Differential effects of temporal pole resection with amygdalohippocampectomy versus selective amygdalohippocampectomy on material-specific memory in patients with mesial temporal lobe epilepsy. Epilepsia 49:88-97, 2008

14. Helmstaedter C, Roeske S, Kaaden S, Elger CE, Schramm $\mathrm{J}$ : Hippocampal resection length and memory outcome in selective epilepsy surgery. J Neurol Neurosurg Psychiatry 82:1375-1381, 2011

15. Hu WH, Zhang C, Zhang K, Meng FG, Chen N, Zhang JG: Selective amygdalohippocampectomy versus anterior temporal lobectomy in the management of mesial temporal lobe epilepsy: a meta-analysis of comparative studies. J Neurosurg 119:1089-1097, 2013

16. Jeong W, Lee H, Kim JS, Chung CK: Neural basis of episodic memory in the intermediate term after medial temporal lobe resection. J Neurosurg 131:790-798, 2019

17. Jones-Gotman M, Zatorre RJ, Olivier A, Andermann F, Cendes F, Staunton H, et al: Learning and retention of words and designs following excision from medial or lateral temporal-lobe structures. Neuropsychologia 35:963-973, 1997

18. Kim YH, Kim CH, Kim JS, Lee SK, Chung CK: Resection frequency map after awake resective surgery for non-lesional neocortical epilepsy involving eloquent areas. Acta Neurochir (Wien) 153:1739-1749, 2011

19. Kivisaari SL, Probst A, Taylor KI: The perirhinal, entorhinal, and parahippocampal cortices and hippocampus: an overview of functional anatomy and protocol for their segmentation in MR images, in Ulmer S, Jansen O (eds): fMRI. Berlin: Springer, 2013, pp 239-267

20. Liu A, Thesen T, Barr W, Morrison C, Dugan P, Wang X, et al: Parahippocampal and entorhinal resection extent predicts verbal memory decline in an epilepsy surgery cohort. J Cogn Neurosci 29:869-880, 2017

21. Malikova H, Kramska L, Vojtech Z, Sroubek J, Lukavsky J, Liscak R: Relationship between remnant hippocampus and amygdala and memory outcomes after stereotactic surgery for mesial temporal lobe epilepsy. Neuropsychiatr Dis Treat 11:2927-2933, 2015

22. Morino M, Uda T, Naito K, Yoshimura M, Ishibashi K, Goto $\mathrm{T}$, et al: Comparison of neuropsychological outcomes after selective amygdalohippocampectomy versus anterior temporal lobectomy. Epilepsy Behav 9:95-100, 2006

23. Nemanic S, Alvarado MC, Bachevalier J: The hippocampal/ parahippocampal regions and recognition memory: insights from visual paired comparison versus object-delayed nonmatching in monkeys. J Neurosci 24:2013-2026, 2004

24. Noulhiane M, Piolino P, Hasboun D, Clemenceau S, Baulac M, Samson S: Autobiographical memory after temporal lobe resection: neuropsychological and MRI volumetric findings. Brain 130:3184-3199, 2007

25. Nunn JA, Graydon FJ, Polkey CE, Morris RG: Differential spatial memory impairment after right temporal lobectomy demonstrated using temporal titration. Brain 122:47-59, 1999

26. Pascalis $\mathrm{O}$, Bachevalier $\mathrm{J}$ : Neonatal aspiration lesions of the hippocampal formation impair visual recognition memory when assessed by paired-comparison task but not by delayed nonmatching-to-sample task. Hippocampus 9:609-616, 1999

27. Rorden C, Bonilha L, Fridriksson J, Bender B, Karnath HO: Age-specific CT and MRI templates for spatial normalization. Neuroimage 61:957-965, 2012 
28. Saling MM: Verbal memory in mesial temporal lobe epilepsy: beyond material specificity. Brain 132:570-582, 2009

29. Schramm J: Temporal lobe epilepsy surgery and the quest for optimal extent of resection: a review. Epilepsia 49:12961307, 2008

30. Sherman EM, Wiebe S, Fay-McClymont TB, Tellez-Zenteno J, Metcalfe A, Hernandez-Ronquillo L, et al: Neuropsychological outcomes after epilepsy surgery: systematic review and pooled estimates. Epilepsia 52:857-869, 2011

31. Shin MS, Lee S, Seol SH, Lim YJ, Park EH, Sergeant JA, et al: Changes in neuropsychological functioning following temporal lobectomy in patients with temporal lobe epilepsy. Neurol Res 31:692-701, 2009

32. Silbergeld DL: Extent of medial temporal resection on outcome from anterior temporal lobectomy: a randomized prospective study. Neurosurgery 41:327-328, 1997

33. Skirrow C, Cross JH, Harrison S, Cormack F, Harkness W, Coleman R, et al: Temporal lobe surgery in childhood and neuroanatomical predictors of long-term declarative memory outcome. Brain 138:80-93, 2015

34. Smith EE, Salat DH, Jeng J, McCreary CR, Fischl B, Schmahmann JD, et al: Correlations between MRI white matter lesion location and executive function and episodic memory. Neurology 76:1492-1499, 2011

35. Spencer D, Burchiel K: Selective amygdalohippocampectomy. Epilepsy Res Treat 2012:382095, 2012

36. Squire LR, Zola-Morgan S: The medial temporal lobe memory system. Science 253:1380-1386, 1991

37. Tanriverdi T, Dudley RW, Hasan A, Al Jishi A, Al Hinai Q, Poulin N, et al: Memory outcome after temporal lobe epilepsy surgery: corticoamygdalohippocampectomy versus selective amygdalohippocampectomy. J Neurosurg 113:1164-1175, 2010

38. Trenerry MR, Jack CR Jr, Ivnik RJ, Sharbrough FW, Cascino GD, Hirschorn KA, et al: MRI hippocampal volumes and memory function before and after temporal lobectomy. Neurology 43:1800-1805, 1993

39. Vaz SA: Nonverbal memory functioning following right anterior temporal lobectomy: a meta-analytic review. Seizure 13:446-452, 2004
40. Willment KC, Golby A: Hemispheric lateralization interrupted: material-specific memory deficits in temporal lobe epilepsy. Front Hum Neurosci 7:546, 2013

41. Wolf RL, Ivnik RJ, Hirschorn KA, Sharbrough FW, Cascino GD, Marsh WR: Neurocognitive efficiency following left temporal lobectomy: standard versus limited resection. J Neurosurg 79:76-83, 1993

42. Yonelinas AP, Otten LJ, Shaw KN, Rugg MD: Separating the brain regions involved in recollection and familiarity in recognition memory. J Neurosci 25:3002-3008, 2005

\section{Disclosures}

The authors report no conflict of interest concerning the materials or methods used in this study or the findings specified in this paper.

\section{Author Contributions}

Conception and design: Chung, D Kim. Acquisition of data: Shin. Analysis and interpretation of data: Chung, D Kim, JS Kim.

Drafting the article: D Kim. Critically revising the article: Chung, Jeong. Reviewed submitted version of manuscript: Chung, D Kim, JS Kim, Jeong. Approved the final version of the manuscript on behalf of all authors: Chung. Statistical analysis: D Kim, JS Kim. Study supervision: Chung.

\section{Supplemental Information \\ Previous Presentations}

Portions of this work were presented in abstract form as proceedings at the 24th Korean Epilepsy Congress, Seoul, Republic of Korea, June 15, 2019, and the 19th European Congress of Neurosurgery, Dublin, Ireland, September 27, 2019.

\section{Correspondence}

Chun Kee Chung: Seoul National University Hospital, Seoul, Republic of Korea.chungc@snu.ac.kr. 